\title{
Optimal Control Based on the Polynomial Least Squares Method
}

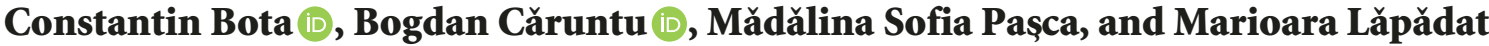 \\ Department of Mathematics "Politehnica" University of Timişoara, P-ta Victoriei, 2, Timişoara 300006, Romania \\ Correspondence should be addressed to Constantin Bota; constantin.bota@upt.ro
}

Received 23 August 2018; Accepted 17 October 2018; Published 28 October 2018

Guest Editor: Hiroaki Mukaidani

Copyright (C) 2018 Constantin Bota et al. This is an open access article distributed under the Creative Commons Attribution License, which permits unrestricted use, distribution, and reproduction in any medium, provided the original work is properly cited.

In this paper an approach for computing an optimal control law based on the Polynomial Least Squares Method (PLSM) is presented. The initial optimal control problem is reformulated as a variational problem whose corresponding Euler-Lagrange equation is solved by using PLSM. A couple of examples emphasize the accuracy of the method.

\section{Introduction}

Optimal control problems occur in many areas of science and engineering such as system mechanics, hydrodynamics, elasticity theory, geometrical optics, and aerospace engineering, and they are one of the several applications and extensions of the calculus of variations.

The beginning of optimal control is represented by the Brachistochrone problem formulated by Galileo in 1683: A mass material point $\mathrm{m}$ moves without friction along a vertical curve joining the points $\left(x_{0}, y_{0}\right)$ and $\left(x_{1}, y_{1}\right)$. There is the question of finding such a curve for which the scroll time is minimal, curve called brachistochrone. Galileo's attempts to resolve it were incorrect $[1,2]$. The problem raised great interest at that time and solutions were proposed by many mathematicians like Bernoulli, Leibnitz, l'Hopital, and Newton [1]. These results were published by Euler in 1744, who concluded "nothing at all takes place in the universe in which some rule of maximum or minimum does not appear." Euler also formulated the problem in general terms as the problem of finding the curve $y(t)$ over the interval $[a, b]$ (with $y(a)$ and $y(b)$ known) which minimizes:

$$
J=\int_{a}^{b} F\left(t, y(t), y^{\prime}(t)\right) d t
$$

for some given function $F\left(t, y(t), y^{\prime}(t)\right)$, where $y^{\prime}=d y / d t$.

Euler presented a necessary condition of optimality for the curve $y(t)$ :

$$
\frac{d}{d t} F_{y^{\prime}}\left(t, y(t), y^{\prime}(t)\right)=F_{y}\left(t, y(t), y^{\prime}(t)\right)
$$

where $F_{y^{\prime}}$ and $F_{y}$ represent the partial derivatives with respect to $y^{\prime}$ and $y$, respectively.

The solution techniques proposed initially had been of a geometric nature until 1755 when Lagrange described an analytical approach, based on perturbations or "variations" of the optimal curve and using his "undetermined multipliers," which led directly to Euler's necessary condition, now known as the "Euler-Lagrange equation." Euler also adopted this approach and renamed the subject "the calculus of variations" [3].

In the years to come, considerable efforts have been made to develop optimal control techniques. A classification of methods for solving optimal control problems is presented by Berkani et al. in [3]. Among the most used ones we mention the following.

(i) The Dynamic Programming method, based on the principle of optimality, was first formulated by Bellman [4] and often used in the analysis and design of automatic control systems. Bellman's partial differential equation and the boundary conditions included are necessary conditions for obtaining the minimum of the optimal control problem.

(ii) The Pontryagin Minimum Principle [5] is built on defining the Hamiltonian function by introducing adjoint variables. The optimal control law is obtained by solving the canonical differential equations (the Hamilton equations) which are the necessary conditions of optimality according to the minimum principle [6]. The optimality conditions are in general not able to provide the exact optimum since the resulting two-point boundary value problem (Bellman partial differential equation) is not easy to be solved 
analytically and usually computational methods are employed [7-9].

In this paper we apply the Polynomial Least Squares Method (PLSM) in order to compute approximate analytical polynomial solutions for a optimal control problems. This method was used by C. Bota and B. Căruntu in 2014 to compute approximate analytical solutions for the Brusselator system which is a fractional-order system of nonlinear differential equations [10]. In the following years the accuracy of the method is emphasized by its use in solving several types of differential equations [11-13].

The optimal control problem approached in this paper is the computation of the optimal control law $u(t):\left[0, t_{f}\right] \subset$ $\mathscr{R} \longrightarrow \mathscr{R}$ which minimizes the performance index:

$$
J=\int_{0}^{t_{f}} F(y(t), u(t), t) d t
$$

where the state equation is

$$
y^{\prime}(t)=f(y(t), u(t), t)
$$

and the state variable $y(t)$ satisfies the constraints $y(0)=y_{0}$ and $y\left(t_{f}\right)=y_{f}$.

We will assume that $\mathrm{F}$ is of class $C^{1}$, so the solution of the optimal control problem exists and is unique for the given conditions. The state equation (4) may be linear or nonlinear but we also assume that $u(t)$ can be explicitly obtained from (4) as a function of $y(t)$. In this case solving the optimal control problem is equivalent to solving the variational problem of finding the minimum of functional:

$$
J=\int_{0}^{t_{f}} G\left(t, y(t), y^{\prime}(t)\right) d t
$$

with

$$
\begin{gathered}
y(0)=y_{0}, \\
y\left(t_{f}\right)=y_{f}
\end{gathered}
$$

where the relation (5) is obtained from (3) by substituting the expression for $u(t)$ as a function of $y(t)(4)$.

The necessary condition for the uniqueness of the solution to the problem (5)-(6) is that $y(t)$ satisfies the conditions (6) and the Euler-Lagrange equation:

$$
\frac{\partial G\left(t, y(t), y^{\prime}(t)\right)}{\partial y(t)}=\frac{d}{d t}\left(\frac{\partial G\left(t, y(t), y^{\prime}(t)\right)}{\partial y^{\prime}(t)}\right) .
$$

\section{Approximate Solution for an Optimal Control Problem Using the Polynomial Least Squares Method}

2.1. The Polynomial Least Squares Method. The EulerLagrange equation associated with the optimal problem (5)(6) may have the expression:

$$
y^{\prime \prime}(t)=\mathscr{F}\left(y^{\prime}(t), y(t), t\right)
$$

where $\mathscr{F}$ may be linear or nonlinear. We associate with this equation the following operator:

$$
D(y(t))=y^{\prime \prime}(t)-\mathscr{F}\left(y^{\prime}(t), y(t), t\right)
$$

If we denote by $\tilde{y}(t)$ an approximate solution of (8), the error obtained by replacing the exact solution $y(t)$ with the approximation $\tilde{y}(t)$ is given by the remainder:

$$
\mathscr{R}(t, \tilde{y}(t))=D(\tilde{y}(t)), \quad t \in\left[0, t_{f}\right] .
$$

Taking into account the boundary conditions (6), for $\epsilon \in R+$, we will compute approximate polynomial solutions $\tilde{y}$ of the problem $(8),(6)$ on the interval $\left[0, t_{f}\right]$ as follows.

Definition 1. We call an $\epsilon$-approximate polynomial solution of the problem (8), (6) an approximate polynomial solution $\tilde{y}$ satisfying the relations

$$
\begin{aligned}
|\mathscr{R}(t, \tilde{y})| & <\epsilon \\
\tilde{y}(0) & =y_{0}, \\
\tilde{y}\left(t_{f}\right) & =y_{f}
\end{aligned}
$$

We call a weak $\epsilon$-approximate polynomial solution of the problem (8), (6) an approximate polynomial solution $\tilde{y}$ satisfying the relation

$$
\int_{0}^{t_{f}}|\mathscr{R}(t, \tilde{y})| d t \leq \epsilon
$$

together with the initial conditions (6)

Definition 2. Let $P_{m}(t)=c_{0}+c_{1} t+c_{2} t^{2}+\cdots+c_{m} t^{m}, c_{i} \in \mathbb{R}$, $i=0,1, \ldots, m$, be a sequence of polynomials satisfying the conditions $P_{m}(0)=y_{0}, P_{m}\left(t_{f}\right)=y_{f}$.

We call the sequence of polynomials $P_{m}(t)$ convergent to the solution of the problem (8), (6) if $\lim _{m \rightarrow \infty} D\left(P_{m}(t)\right)=0$.

We observe that from the hypothesis of the initial problem (8), (6) it follows that there exists a sequence of polynomials $P_{m}(t)$ which converges to the solution of the problem. We will compute a weak $\epsilon$-approximate polynomial solution, in the sense of the Definition 1, of the type

$$
\tilde{y}(t)=\sum_{k=0}^{m} d_{k} t^{k}
$$

where $d_{0}, d_{1}, \ldots, d_{m}$ are constants which are calculated using the following steps:

(i) By substituting the approximate solution (14) in (8) we obtain the remainder:

$$
\mathscr{R}(t, \tilde{y})=\tilde{y}^{\prime \prime}(t)-\mathscr{F}\left(\tilde{y}^{\prime}(t), \tilde{y}(t), t\right)
$$

We remark that if we could find $d_{0}, d_{1}, \ldots, d_{m}$ such that $\mathscr{R}(t, \tilde{y})=0, \tilde{y}(0)=y_{0}, \tilde{y}\left(t_{f}\right)=y_{f}$, then by substituting $d_{0}, d_{1}, \ldots, d_{m}$ in (14) we would obtain the exact solution of the problem (8), (6). This is not generally possible, unless the exact solution is actually a polynomial 
(ii) We attach to the problem (8), (6) the following functional:

$$
\mathscr{J}\left(d_{2}, d_{3} \cdots, d_{m}\right)=\int_{0}^{t_{f}} \mathscr{R}^{2}(t, \tilde{y}(t)) d t
$$

where $d_{0}, d_{1}$ are computed as functions of $d_{2}$, $d_{3} \cdots d_{m}$ using the conditions (6).

(iii) We compute the values $d_{2}^{0}, d_{3}^{0}, \cdots d_{m}^{0}$ as the values which give the minimum of the functional $\mathscr{J}$ and the values of $d_{0}^{0}$ and $d_{1}^{0}$ as functions of $d_{2}^{0}, d_{3}^{0}, \cdots d_{m}^{0}$ using the conditions (6).

(iv) Using the constants $d_{2}^{0}, d_{3}^{0}, \cdots d_{m}^{0}$ previously determined we compute the polynomial

$$
M_{m}(t)=\sum_{k=0}^{m} d_{k}^{0} t^{k}
$$

Theorem 3. The sequence of polynomials $M_{m}(t)$ from (17) satisfies the property

$$
\lim _{t \rightarrow \infty} \int_{0}^{t_{f}} \mathscr{R}^{2}\left(t, M_{m}(t)\right) d t=0
$$

Moreover, if $\forall \epsilon>0, \exists m_{o} \in \mathbf{N}, m>m_{0}$, it follows that $M_{m}(t)$ is a weak $\epsilon$-approximate polynomial solution of the problem (8), (6)

Proof. Based on the way the polynomials $M_{m}(t)$ are computed and taking into account the relations (15)-(17), the following inequalities are satisfied:

$$
0 \leq \int_{0}^{t_{\mathrm{f}}} \mathscr{R}^{2}\left(t, M_{m}(t)\right) d t \leq \int_{0}^{t_{f}} \mathscr{R}^{2}\left(t, P_{m}(t)\right) d t,
$$

$\forall m \in \mathbf{N}$

where $P_{m}(t)$ is the sequence of polynomials introduced in Definition 2.

It follows that

$$
\begin{aligned}
0 & \leq \lim _{t \rightarrow \infty} \int_{0}^{t_{f}} \mathscr{R}^{2}\left(t, M_{m}(t)\right) d t \\
& \leq \lim _{t \rightarrow \infty} \int_{0}^{t_{f}} \mathscr{R}^{2}\left(t, P_{m}(t)\right) d t=0 .
\end{aligned}
$$

We obtain

$$
\lim _{t \longrightarrow \infty} \int_{a}^{b} \mathscr{R}^{2}\left(t, M_{m}(t)\right) d t=0 .
$$

From this limit we obtain that $\forall \epsilon>0, \exists m_{0} \in \mathbf{N}, m>m_{0}$ and it follows that $M_{m}(t)$ is a weak $\epsilon$-approximate polynomial solution of the problem (8), (6).

Remark 4. In order to find $\epsilon$-approximate polynomial solutions of the problem (8), (6) by using the Polynomial Least Squares Method we will first determine weak approximate polynomial solutions, $\tilde{y}$. If $|\mathscr{R}(t, \tilde{y})|<\epsilon$ then $\tilde{y}$ is also an $\epsilon-$ approximate polynomial solution of the problem.
2.2. Application of the Polynomial Least Squares Method for an Optimal Control Problem. We will find the approximate solution of the optimal control problem (3)-(4) using the following steps:

(i) We transform the optimal control problem (3)-(4) in a variational problem (5)-(6) as described in the introduction.

(ii) We attach to the variational problem (5)-(6) the corresponding Euler-Lagrange equation (7), (8).

(iii) We compute the approximate solution $\tilde{y}(t)$ of the Euler-Lagrange equation using PLSM as described in the previous section. Thus $\tilde{y}(t)$ is an approximation of the state variable $y(t)$ of the optimal control problem.

(iv) Finally we compute an approximation $\widetilde{u}(t)$ of the optimal control law $u(t)$ by means of the state equation (4).

\section{Applications}

In this section we apply the Polynomial Least Squares Method in order to compute analytical approximate optimal control laws for three optimal control problems.

3.1. Application 1. We consider the following optimal control problem [3]:

$$
\min _{u(t)} \int_{0}^{1}\left[(2-y(t))^{2}+u^{2}(t)\right] d t
$$

where the state equation is

$$
y^{\prime}(t)=u(t)-\frac{1}{4} \sqrt{y(t)}
$$

and the boundary conditions are

$$
\begin{aligned}
& y(0)=0, \\
& y(1)=2
\end{aligned}
$$

The exact solution of this problem is [3]

$$
\begin{aligned}
& y(t) \\
& =\frac{e^{-t}\left(-e-63 e^{2}-63 e^{t}+63 e^{2 t}+63 e^{2+t}+e^{1+2 t}\right)}{32\left(-1+e^{2}\right)}
\end{aligned}
$$

In order to apply PLSM we follow the steps presented in the previous section:

(i) From the state equation (23) we obtain the optimal control law $u(t)$ as a function of the state variable $y(t)$ :

$$
u(t)=y^{\prime}(t)+\frac{1}{4} \sqrt{y(t)}
$$

Replacing this expression of $u(t)$ in the performance index (22) we transform the initial optimal control problem into the following variational problem: 
(a) Find the minimum of the functional

$$
\int_{0}^{1}\left[(2-y(t))^{2}+\left(y^{\prime}(t)+\frac{1}{4} \sqrt{y(t)}\right)^{2}(t)\right] d t
$$

subject to the boundary conditions (24).

(ii) The corresponding Euler-Lagrange equation is

$$
y^{\prime \prime}(t)-y(t)+\frac{63}{32}=0
$$

(iii) We compute using PLSM an approximate analytical solution of the type

$$
\begin{aligned}
\tilde{y}(t)= & d_{0}+d_{1} \cdot t+d_{2} \cdot t^{2}+d_{3} \cdot t^{3}+d_{4} \cdot t^{4}+d_{5} \cdot t^{5} \\
& +d_{6} \cdot t^{6}+d_{7} \cdot t^{7}+d_{8} \cdot t^{8}+d_{9} \cdot t^{9}
\end{aligned}
$$

From the boundary conditions (24) we obtain $\widetilde{d}_{0}=0$ and $d_{1}=2-d_{2}-d_{3}-d_{4}-d_{5}-d_{6}-d_{7}-d_{8}-d_{9}$.

The corresponding remainder (15) is

$$
\begin{aligned}
& \mathscr{R}(t)=-\frac{63}{16}-2 \cdot\left(2 \cdot d_{2}+6 \cdot d_{3} \cdot t+12 \cdot d_{4} \cdot t^{2}+20\right. \\
& \cdot d_{5} \cdot t^{3}+30 \cdot d_{6} \cdot t^{4}+42 \cdot d_{7} \cdot t^{5}+56 \cdot d_{8} \cdot t^{6} \\
& \left.\quad+72 \cdot d_{9} \cdot t^{7}\right)+2 \\
& \quad\left(\left(2-d_{2}-d_{3}-d_{4}-d_{5}-d_{6}-d_{7}-d_{8}-d_{9}\right) \cdot t\right. \\
& +d_{2} \cdot t^{2}+d_{3} \cdot t^{3}+d_{4} \cdot t^{4}+d_{5} \cdot t^{5}+d_{6} \cdot t^{6}+d_{7} \\
& \left.\cdot t^{7}+d_{8} \cdot t^{8}+d_{9} \cdot t^{9}\right) .
\end{aligned}
$$

By minimizing the functional $(16) \mathcal{J}\left(d_{2}, d_{3} \cdots, d_{9}\right)$ (too large to be included here) we obtain the values for $d_{2}, d_{3} \cdots, d_{9}$. We compute the corresponding values of $d_{0}$ and $d_{1}$ using again the conditions (24) and we replace all these values in $\tilde{y}(t)$ to obtain our approximation:

$$
\begin{aligned}
\tilde{y}(t)= & 2.6116294098342876 \cdot t \\
& +0.9843749991096482 \cdot t^{2} \\
& +0.43527154661696676 \cdot t^{3} \\
& +0.08203105649571096 \cdot t^{4} \\
& +0.021762704663334236 \cdot t^{5} \\
& +0.0027321111314777993 \cdot t^{6} \\
& +0.0005146366961518619 \cdot t^{7} \\
& +0.0000454584242327247 \cdot t^{8} \\
& +0.000005327350329483319 \cdot t^{9} .
\end{aligned}
$$

TABLE 1: Absolute errors of the approximations of the state variable $\tilde{y}(t)$ and the optimal control law $\widetilde{u}(t)$ obtained by using PLSM for Application 1.

\begin{tabular}{lcc}
\hline$t$ & $\tilde{y}(t)$ & $\tilde{u}(t)$ \\
\hline 0 & 0 & $4.440892099 \cdot 10^{-16}$ \\
0.1 & $2.317035452 \cdot 10^{-13}$ & $2.194910920 \cdot 10^{-11}$ \\
0.2 & $6.393774399 \cdot 10^{-13}$ & $1.921662829 \cdot 10^{-11}$ \\
0.3 & $1.423638984 \cdot 10^{-12}$ & $7.726264073 \cdot 10^{-12}$ \\
0.4 & $7.804867863 \cdot 10^{-14}$ & $2.682920552 \cdot 10^{-11}$ \\
0.5 & $1.620037438 \cdot 10^{-12}$ & $1.299405028 \cdot 10^{-12}$ \\
0.6 & $2.264854970 \cdot 10^{-13}$ & $2.589484183 \cdot 10^{-11}$ \\
0.7 & $1.334266031 \cdot 10^{-12}$ & $9.465761508 \cdot 10^{-12}$ \\
0.8 & $6.727951529 \cdot 10^{-13}$ & $1.677147310 \cdot 10^{-11}$ \\
0.9 & $1.718625242 \cdot 10^{-13}$ & $2.004618693 \cdot 10^{-11}$ \\
1.0 & $2.220446049 \cdot 10^{-16}$ & 0 \\
\hline
\end{tabular}

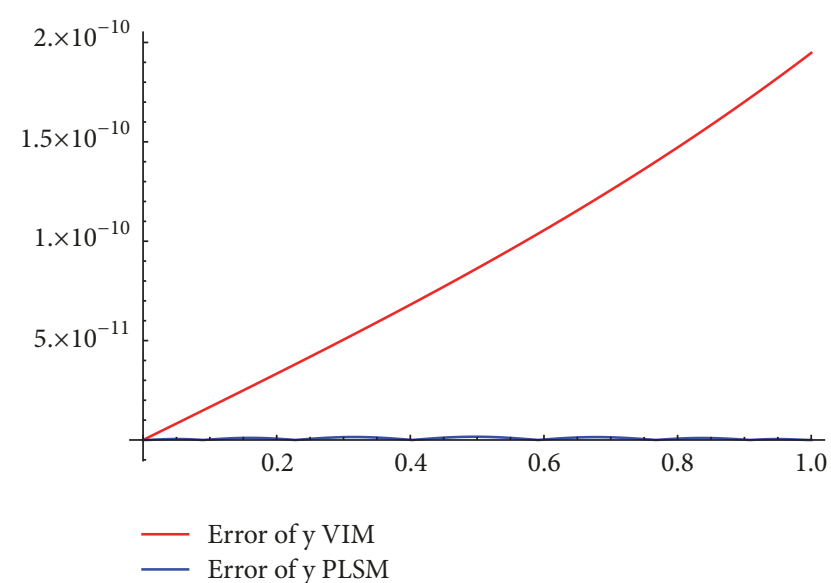

FIgURE 1: The absolute errors corresponding to the approximations of the state variable $\tilde{y}(t)$ in Application 1: approximate solution from [3] given by VIM (red curve) and approximate solution given by PLSM (blue curve).

(iv) Finally we can easily compute an approximation for $\widetilde{u}(t)$ (also not included here because of its large size) by means of (26):

$$
\widetilde{u}(t)=\widetilde{y}^{\prime}(t)+\frac{1}{4} \sqrt{\tilde{y}(t)}
$$

Table 1 presents the absolute errors (as differences in absolute value between the exact value and the approximate one) corresponding to our approximations of the state variable $\tilde{y}(t)$ and of the optimal control law $\tilde{u}(t)$ obtained by using PLSM.

Figures 1 and 2 present the comparison between our results and previous ones computed in [3] by using the Variational Iteration Method (VIM). It can be easily observed that not only is our approximation more precise, but while the error function corresponding to the VIM approximations shows a sizeable increase with $t$, the error function corresponding to PLSM does not. Moreover, another advantage of PLSM is the fact that, evidently, the approximation has the 


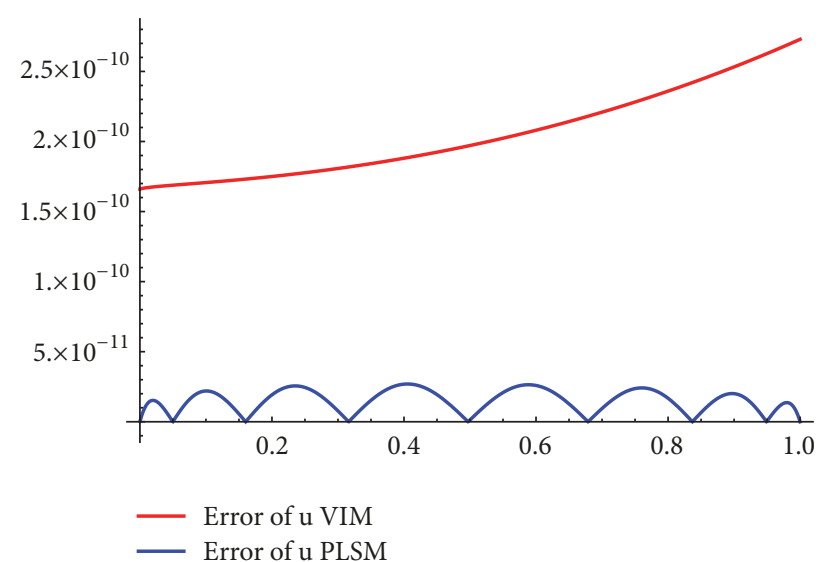

FIGURE 2: The absolute errors corresponding to the approximations of the optimal control law $\widetilde{u}(t)$ in Application 1: approximate solution from [3] given by VIM (red curve) and approximate solution given by PLSM (blue curve).

simplest possible form, namely, a polynomial, and thus is very easy to use in any further computations. Finally, we mention the fact that by increasing the degree of the polynomial $\tilde{y}(t)$ we can obtain higher accuracy: for example, using a 10-th degree polynomial we obtain an overall error of $10^{-14}$.

3.2. Application 2. Our second application is the optimal control problem:

$$
\min _{u(t)} \int_{0}^{1} \frac{1+y^{2}(t)}{u^{2}(t)} d t
$$

where there state equation is

$$
y^{\prime}(t)=u(t)
$$

and the boundary conditions are

$$
\begin{aligned}
& y(0)=0, \\
& y(1)=0.5
\end{aligned}
$$

(i) Replacing the expression of $u(t)$ from (34) in the performance index (33) we obtain the variational problem [6]:

$$
\min _{y(t)} \int_{0}^{1} \frac{1+y^{2}(t)}{y^{\prime 2}(t)} d t
$$

with the same boundary conditions $y(0)=0, y(1)=$ 0.5 .

The exact solution of this problem is [6]

$$
y(t)=\sinh \left(t \cdot \sinh ^{-1}\left(\frac{1}{2}\right)\right)
$$

We apply the same steps as in the previous application:

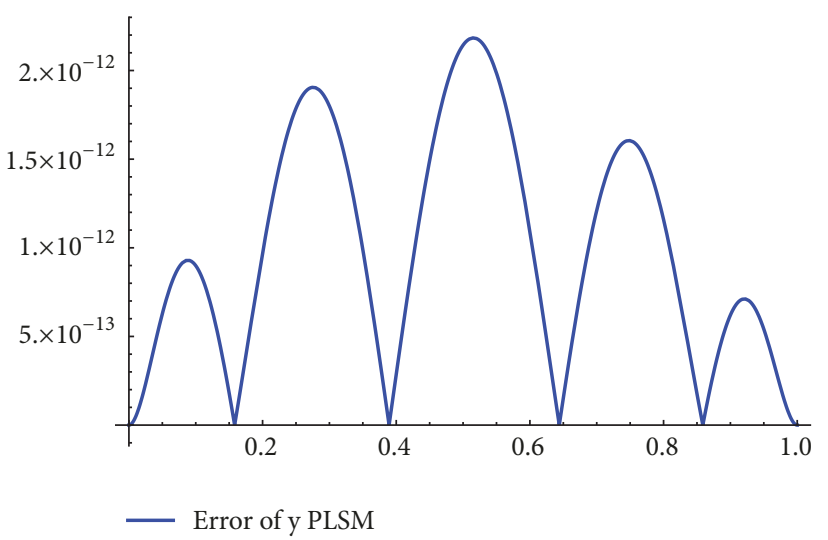

FIGURE 3: The absolute error corresponding to the approximation of the state variable $\widetilde{y}(t)$ in Application 2: approximate solution given by PLSM.

(ii) The corresponding nonlinear Euler Lagrange equation is

$$
y^{\prime \prime}(t)\left(1+y^{2}(t)\right)-y^{\prime}(t) y(t)=0
$$

(iii) We compute using PLSM an approximate analytical solution of the type

$$
\begin{aligned}
\tilde{y}(t)= & d_{0}+d_{1} \cdot t+d_{2} \cdot t^{2}+d_{3} \cdot t^{3}+d_{4} \cdot t^{4}+d_{5} \cdot t^{5} \\
& +d_{6} \cdot t^{6}+d_{7} \cdot t^{7} .
\end{aligned}
$$

From the boundary conditions (35) we obtain $\widetilde{d}_{0}=0$ and $d_{1}=1 / 2-d_{2}-d_{3}-d_{4}-d_{5}-d_{6}-d_{7}$.

We compute again the corresponding reminder (15) and by minimizing the functional (16) $\mathcal{F}\left(d_{2}\right.$, $d_{3} \cdots, d_{7}$ ) we obtain the values for $d_{2} \cdots d_{7}$. Using the conditions (35) and replacing all the values in $\tilde{y}$, our approximation of the state variable is

$$
\begin{aligned}
\widetilde{y}= & 0.4812118250596084 \cdot t-5.310800720418878 \\
& \cdot 10^{-10} \cdot t^{2}+0.018571962082159076 \cdot t^{3} \\
& -3.347040175284603 \cdot 10^{-8} \cdot t^{4} \\
& +0.00021510474426296038 \cdot t^{5} \\
& -8.131321771683893 \cdot 10^{-8} \cdot t^{6} \\
& +1.2234286690670117 \cdot 10^{-6} \cdot t^{7}
\end{aligned}
$$

(iv) Using the state equation (34) we compute an approximation for the optimal control law $\widetilde{u}(t)$.

In Figures 3 and 4 we present the absolute errors corresponding to our approximations of the state variable $\tilde{y}(t)$ and of the optimal control law $\widetilde{u}(t)$ for the problem (33)-(35) obtained by using PLSM. 


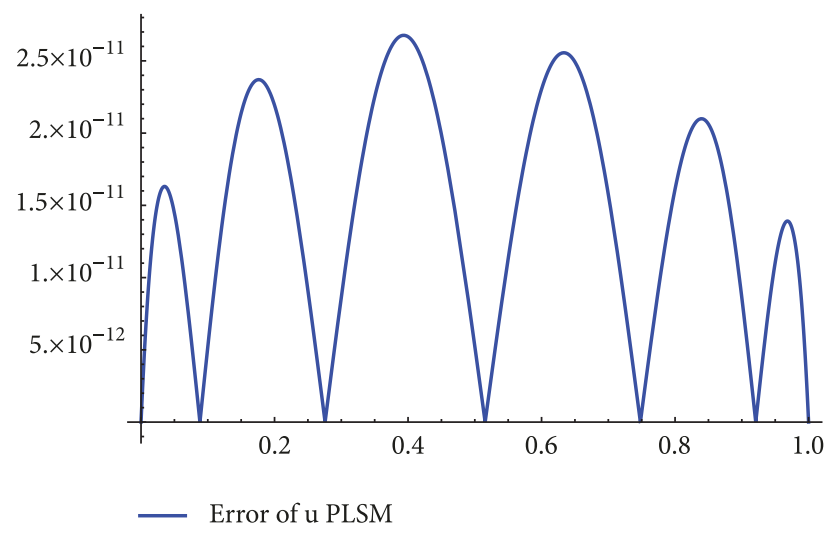

FIGURE 4: The absolute error corresponding to the approximation of the optimal control law $\widetilde{u}(t)$ in Application 2: approximate solution given by PLSM.

3.3. Application 3. Our third application is the well-known linear quadratic regulator (LQR), more precisely the finitehorizon, continuous-time LQR. LQRs have a wide range of applications in engineering such as trajectory tracking and optimization in robotics, control system design for various types of vehicles, automatic voltage regulators in electrical generators, and optimal controls for various types of motors.

The corresponding optimal control problem may be formulated as

$$
\begin{aligned}
J= & \frac{1}{2} x\left(t_{f}\right)^{T} S x\left(t_{f}\right) \\
& +\frac{1}{2} \int_{t_{0}}^{t_{f}}\left(x^{T} P x+2 x^{T} Q u+u^{T} R u\right) d t \\
x^{\prime}(t)= & A x(t)+B u(t) \\
x\left(t_{0}\right)= & x_{0}, \\
x\left(t_{f}\right)= & x_{f}, \\
&
\end{aligned}
$$

We consider the following particular case of the problem (41)-(43) corresponding to the values $\mathrm{A}=1, \mathrm{~B}=1, \mathrm{~S}=8, \mathrm{P}=3$, $\mathrm{Q}=0, \mathrm{R}=1$, and $t_{f}=1[14,15]$ :

The performance index is

$$
J=4 \cdot y^{2}(1)+\frac{1}{2} \int_{0}^{1}\left(3 \cdot y^{2}(t)+u^{2}(t)\right) d t
$$

the state equation is

$$
y^{\prime}(t)=u(t)
$$

and the boundary conditions are

$$
\begin{aligned}
& y(0)=3+\frac{20}{9 e^{4}-5}, \\
& y(1)=8
\end{aligned}
$$

Approximate solutions for this problem were proposed in [14] using the Homotopy Analysis Method and in [15] using the Optimal Homotopy Analysis Method. The exact solution of the problem is

$$
\begin{aligned}
& y(t) \\
& =\frac{e^{-2 t}\left(\left(5+45 e^{2}+72 e^{4}\right) e^{4 t}+e^{2}\left(-40-45 e^{2}+27 e^{4}\right)\right)}{\left(1+e^{2}\right)\left(9 e^{4}-5\right)}
\end{aligned}
$$

The corresponding expression of the control is

$$
\begin{aligned}
& u(t) \\
& =\frac{e^{-2 t}\left(\left(5+45 e^{2}+72 e^{4}\right) e^{4 t}+3 e^{2}\left(40+45 e^{2}-27 e^{4}\right)\right)}{\left(1+e^{2}\right)\left(9 e^{4}-5\right)}
\end{aligned}
$$

Using the same steps presented in the previous examples we computed the following approximation of the state variable:

$$
\begin{aligned}
\tilde{y}(t)= & 3.041119828603783-1.8976676950657474 \\
& \cdot t+6.08223965125759 \cdot t^{2} \\
& -1.2651116099552036 \cdot t^{3} \\
& +2.027411010322069 \cdot t^{4} \\
& -0.2530087549866359 \cdot t^{5} \\
& +0.2702720790557366 \cdot t^{6} \\
& -0.02398312502575098 \\
& \cdot t^{7} 0.01913989850290996 \cdot t^{8} \\
& -0.0011806615481455588 \cdot t^{9} \\
& +0.0007696532612724977 \cdot t^{10} \\
& -0.00002286154490479068 \cdot t^{11} \\
& +0.000022587123028528187 \cdot t^{12}
\end{aligned}
$$

In Figures 5 and 6 we present the absolute errors corresponding to our approximations of the state variable $\tilde{y}(t)$ and of the optimal control law $\widetilde{u}(t)$ for the problem (44)-(46) obtained by using PLSM.

The accuracy of our method is emphasized by a comparison with approximate solutions for Application 3 previously computed by means of other well-known methods. Table 2 presents a comparison of the absolute errors corresponding to the approximations of the state variable $\tilde{y}(t)$ obtained by using the Homotopy Analysis Method (HAM [14]) and by using the Optimal Homotopy Analysis Method (OHAM [15]).

\section{Conclusion}

In this paper the application of the Polynomial Least Squares Method to optimal control problems is presented. 
TABLE 2: Comparison of the absolute errors corresponding to the approximations of the state variable $\tilde{y}(t)$ obtained by using HAM, OHAM, and PLSM for Application 3.

\begin{tabular}{lccc}
\hline$t$ & $\widetilde{y}(t)_{\text {HAM }}$ & $\tilde{y}(t)_{\text {OHAM }}$ & $\tilde{y}(t)_{P L S M}$ \\
\hline 0 & $3.8034 \cdot 10^{-4}$ & $2.2926 \cdot 10^{-7}$ & $4.4409 \cdot 10^{-16}$ \\
0.2 & $3.7677 \cdot 10^{-4}$ & $8.7067 \cdot 10^{-7}$ & $1.7306 \cdot 10^{-12}$ \\
0.4 & $1.2227 \cdot 10^{-4}$ & $3.1004 \cdot 10^{-7}$ & $3.8681 \cdot 10^{-12}$ \\
0.6 & $1.5453 \cdot 10^{-4}$ & $6.0923 \cdot 10^{-8}$ & $9.6811 \cdot 10^{-13}$ \\
0.8 & $2.1309 \cdot 10^{-4}$ & $7.4523 \cdot 10^{-9}$ & $1.6227 \cdot 10^{-12}$ \\
1.0 & 0 & $3.7303 \cdot 10^{-14}$ & 0 \\
\hline
\end{tabular}

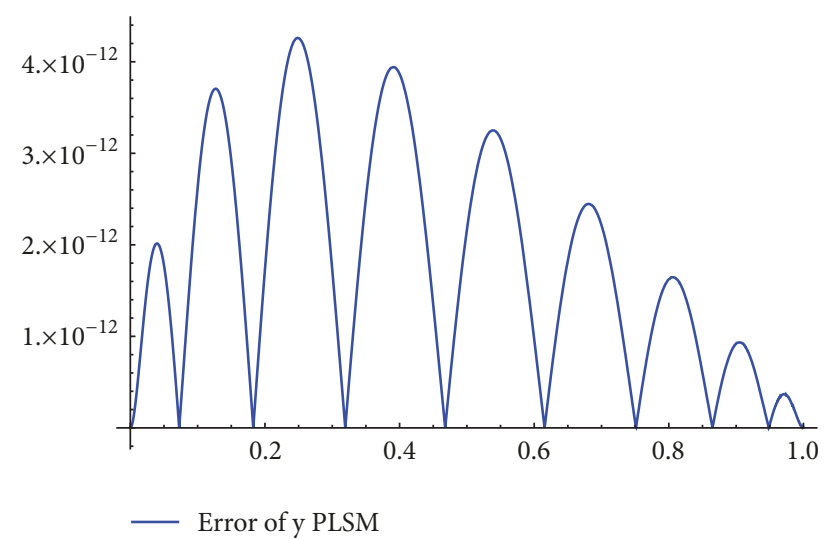

FIGURE 5: The absolute error corresponding to the approximation of the state variable $\tilde{y}(t)$ in Application 3: approximate solution given by PLSM.

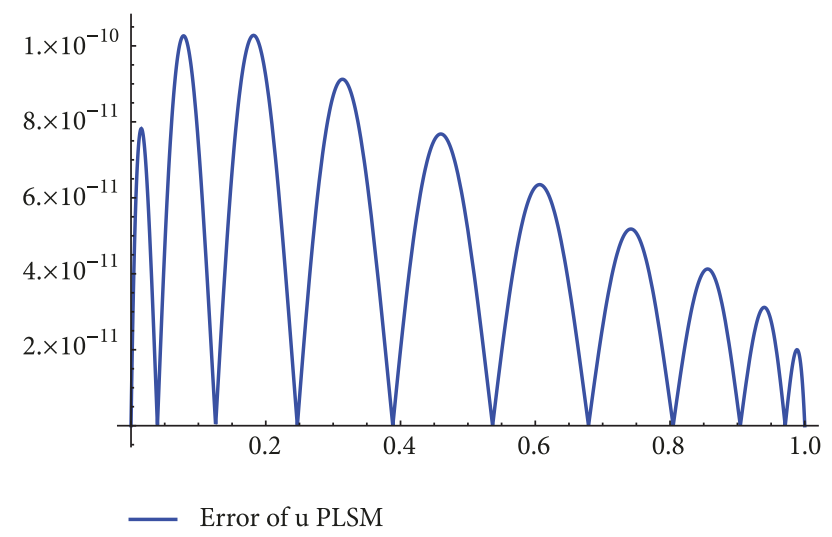

FIGURE 6: The absolute error corresponding to the approximation of the optimal control law $\widetilde{u}(t)$ in Application 3: approximate solution given by PLSM.

In order to apply PLSM the optimal problem is transformed to a variational problem by substituting in the performance index the expression of the control variable given by the state equation. PLSM is able to find accurate approximations of the state variable by computing approximate analytical polynomial solutions of the Euler-Lagrange equation corresponding to the variational problem. The optimal control law is then computed by using the state equation.
The numerical examples included clearly illustrate the accuracy of the method by means of a comparison with solutions previously computed by other methods.

\section{Data Availability}

The data used to support the findings of this study are included within the article.

\section{Conflicts of Interest}

The authors declare that they have no conflicts of interest.

\section{References}

[1] R. W. Sargent, “Optimal control," Journal of Computational and Applied Mathematics, vol. 124, no. 1-2, pp. 361-371, 2000.

[2] H. J. Sussmann and J. C. Willems, "300 years of optimal control: from the brachystochrone to the maximum principle," IEEE Control Systems Magazine, vol. 17, no. 3, pp. 32-44, 1997.

[3] S. Berkani, F. Manseur, and A. Maidi, "Optimal control based on the variational iteration method," Computers \& Mathematics with Applications. An International Journal, vol. 64, no. 4, pp. 604-610, 2012.

[4] R. Luus, "On the application of iterative dynamic programming to singular optimal control problems," Institute of Electrical and Electronics Engineers Transactions on Automatic Control, vol. 37, no. 11, pp. 1802-1806, 1992.

[5] M. J. Sewell, Maximum and Minimum Principles. A Unified Approach, with Applications, Cambridge University Press, New York, NY, USA, 1987.

[6] M. Tatari and M. Dehghan, "Solution of problems in calculus of variations via He's variational iteration method," Physics Letters A, vol. 362, no. 5-6, pp. 401-406, 2007.

[7] B. van Brunt, The Calculus of Variations, Springer, New York, NY, USA, 2004.

[8] J.-H. He, "Variational iteration method for autonomous ordinary differential systems," Applied Mathematics and Computation, vol. 114, no. 2-3, pp. 115-123, 2000.

[9] I. Kucuk, "Active Optimal Control of the KdV Equation Using the Variational Iteration Method," Mathematical Problems in Engineering, vol. 2010, Article ID 929103, 10 pages, 2010.

[10] C. Bota and B. Căruntu, "Approximate Analytical Solutions of the Fractional-Order Brusselator System Using the Polynomial Least Squares Method," Advances in Mathematical Physics, vol. 2015, Article ID 450235, 5 pages, 2015.

[11] C. Bota and B. Caruntu, "Analytic approximate solutions for a class of variable order fractional differential equations using 
the polynomial least squares method," Fractional Calculus and Applied Analysis, vol. 20, no. 4, pp. 1043-1050, 2017.

[12] C. Bota and B. Caruntu, "Analytical approximate solutions for quadratic Riccati differential equation of fractional order using the polynomial least squares method," Chaos, Solitons \& Fractals, vol. 102, pp. 339-345, 2017.

[13] B. Caruntu and C. Bota, "Approximate Analytical Solutions of the Regularized Long Wave Equation Using the Optimal Homotopy Perturbation Method," The Scientific World Journal, vol. 201, Article ID 721865, 6 pages, 2014.

[14] M. S. Zahedi and H. S. Nik, "On homotopy analysis method applied to linear optimal control problems," Applied Mathematical Modelling: Simulation and Computation for Engineering and Environmental Systems, vol. 37, no. 23, pp. 9617-9629, 2013.

[15] W. Jia, X. He, and L. Guo, "The optimal homotopy analysis method for solving linear optimal control problems," Applied Mathematical Modelling: Simulation and Computation for Engineering and Environmental Systems, vol. 45, pp. 865-880, 2017. 


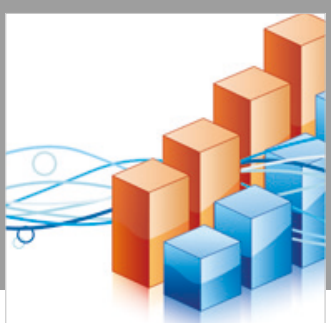

Advances in

Operations Research

\section{-n-m}
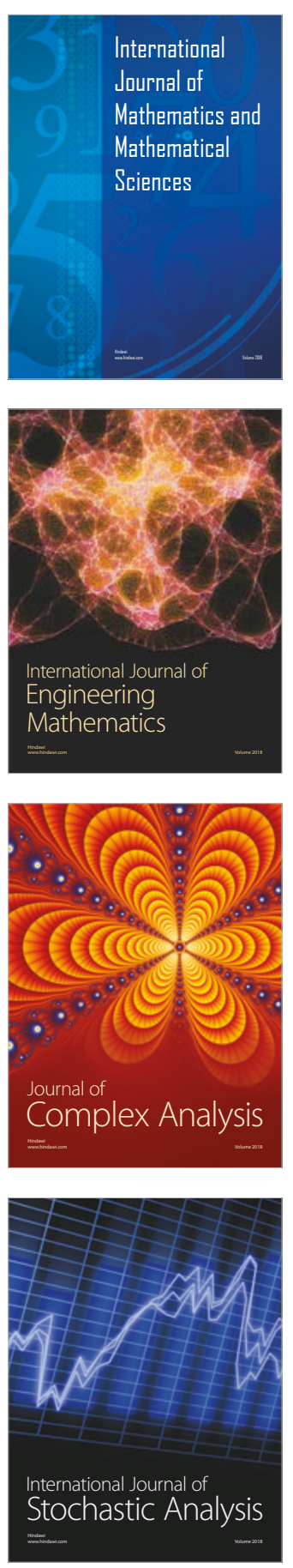
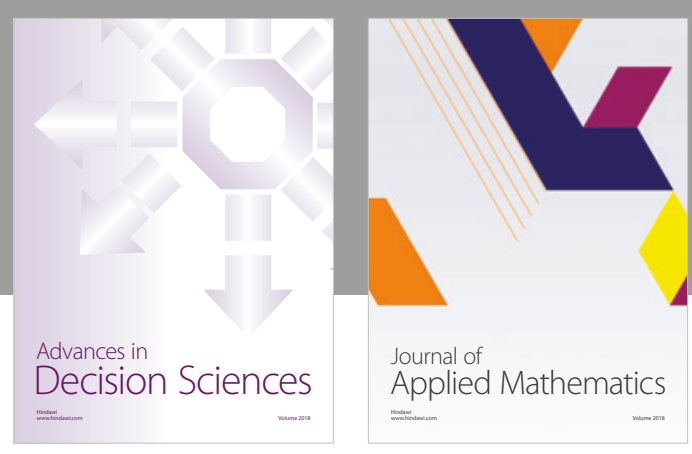

Journal of

Applied Mathematics
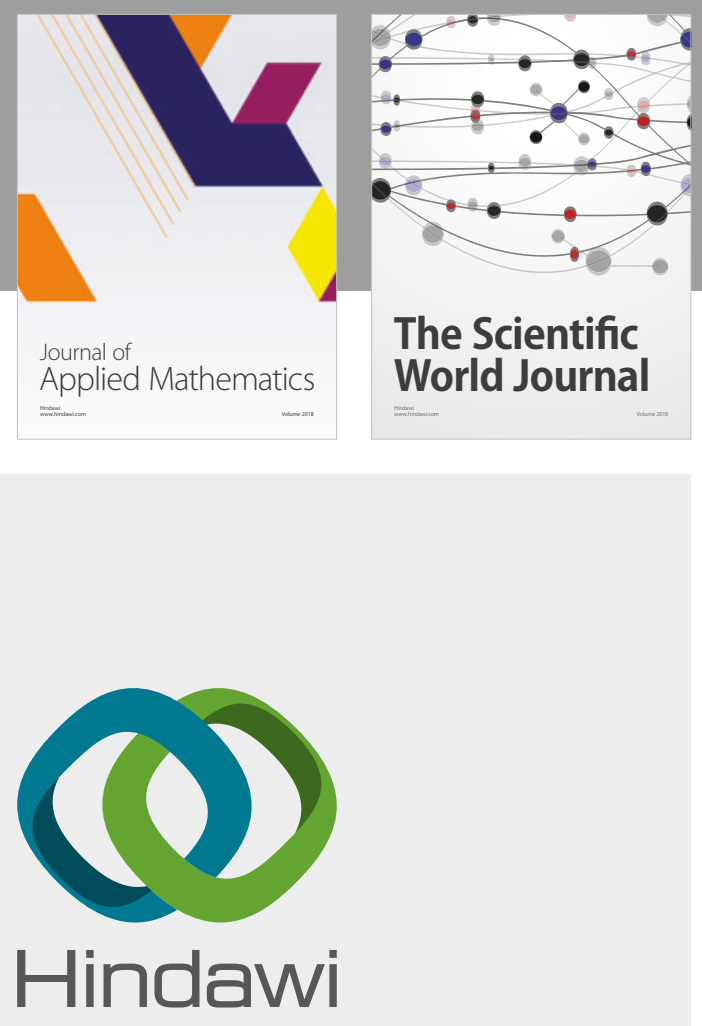

Submit your manuscripts at

www.hindawi.com

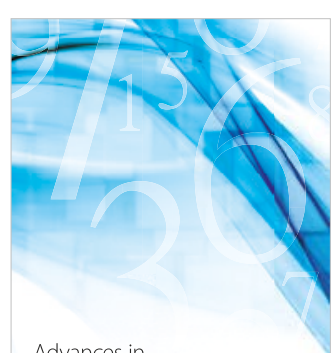

Advances in
Numerical Analysis
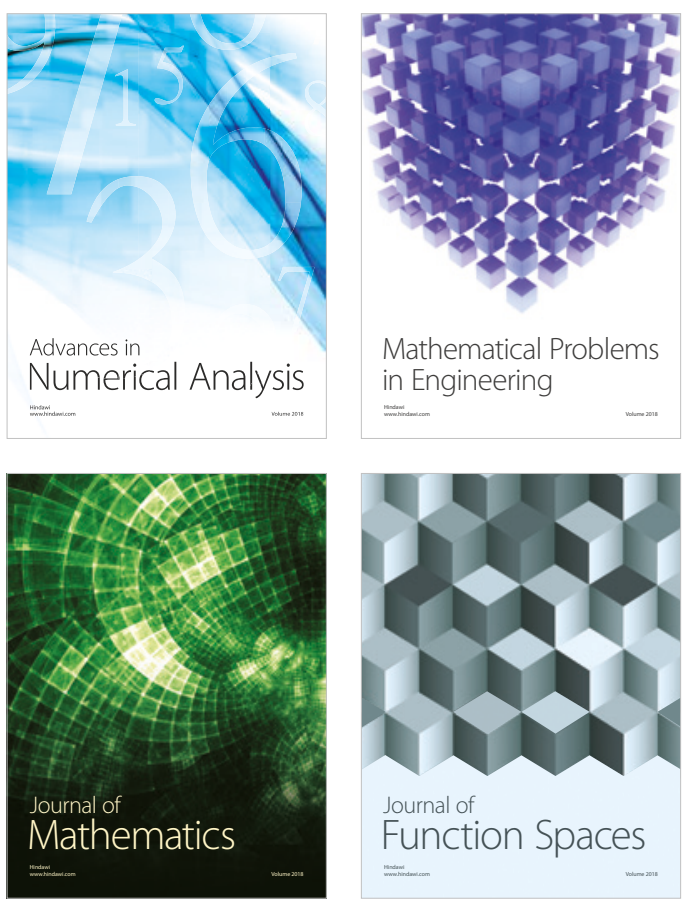

Mathematical Problems in Engineering

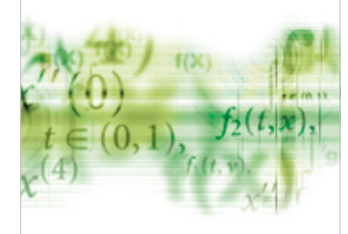

International Journal of

Differential Equations

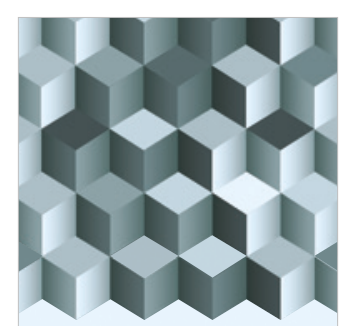

Journal of

Function Spaces

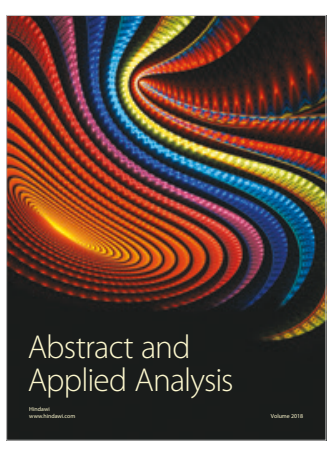

The Scientific

World Journal

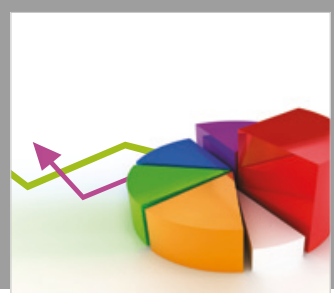

Journal of

Probability and Statistics
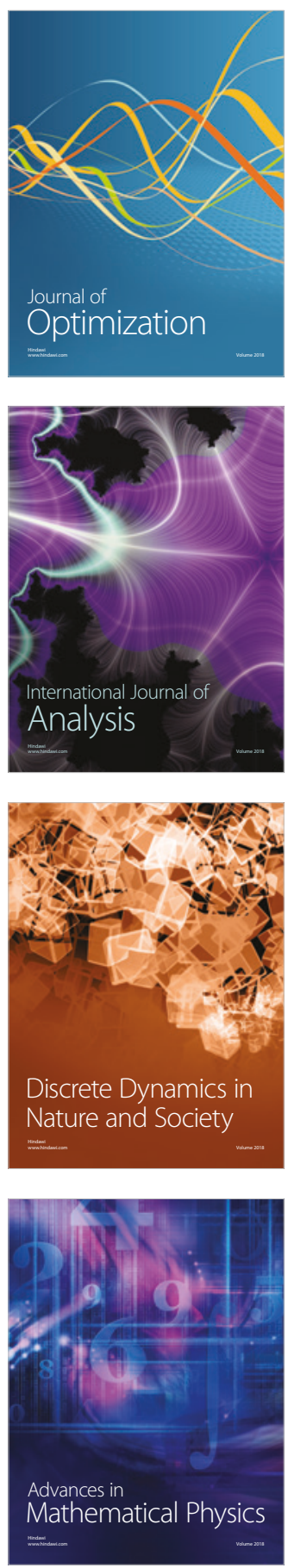